\title{
ELECTRON TRANSPORT PROPERTIES OF UAsSe
}

\author{
Z. Henkie, R. Fabrowski and A. Wojakowski \\ W. Trzebiatowski Institute of Low Temperature and Structure Research \\ Polish Academy of Sciences \\ P.O. Box 937, 50-950 Wrocław 2, Poland
}

\begin{abstract}
Anisotropy of resistivity $(2-450 \mathrm{~K})$, in-plane thermoelectric power $(4-300 \mathrm{~K})$ and Hall's constants $(200-450 \mathrm{~K})$ for UAsSe were determined. It is highly anisotropic, uniaxial ferromagnet below $T_{\mathrm{C}}=113 \mathrm{~K}$. The compound is classified as Kondo lattice system becoming noncoherent below $\approx 0.5 T_{\mathrm{C}}$.
\end{abstract}

PACS numbers: 72.15.-v, 72.15.Qm, 75.50.Cc

There is a great interest in some group of uranium and cerium compounds consisting of increasing number of members subsequently termed as anomalous, intermediate valence, Kondo's lattice, and heavy fermions systems. This group has been extended to systems whose well-known members are CeNiSn and $\mathrm{Ce}_{3} \mathrm{Bi}_{4} \mathrm{Pt}_{3}$ compounds. They are termed as hybridization gap - or semiconducting mixed valence - compounds $[1,2]$. We will show that some features of electron transport behaviour of UAsSe correspond to the systems mentioned above.

The UAsSe crystallizes in tetragonal $(P 4 / \mathrm{nmm})$ structure. It becomes uniaxial and highly anisotropic ferromagnet below $113 \mathrm{~K}$ with spontaneous magnetic moment along $c$-axis [3]. Its temperature dependence of in-plane $(\perp c)$ resistivity $(\rho(300)=280 \mu \Omega \mathrm{cm})$ and magnetoresistivity are already known $[4,5]$. We will present the temperature variation of resistivity anisotropy, in-plane thermoelectric power and the Hall coefficient.

Plate-like single crystals of UAsSe, of thickness up to $0.5 \mathrm{~mm}$ and other dimensions up to $10 \mathrm{~mm}$, were grown by chemical vapor transport method. The IIall coefficient as well as the thermoelectric power were measured by conventional dc methods on samples whose length exceeds the width more than three times. The resistivity anisotropy was measured by Montgomery's modification of the van der Pauw method on rectangular sample with dimensions of $0.83 \times 0.25 \times 0.29 \mathrm{~mm}^{3}$ along $a-, b$ - and $c$-axes, respectively. Four silver wires were soldered with indium to the sample corners in the ac plane to determine the resistivity tensors along $a$ and $c$-axis.

Temperature dependence of the resistivity tensors is shown in Fig. 1. The Curie temperature, $T_{\mathrm{C}}$, is equal to $102 \pm 0.5 \mathrm{~K}$ for this crystal. It was determined by the maximum of $\mathrm{d} \rho_{a} / \mathrm{d} T$. It can be compared with $T_{\mathrm{C}}=109 \mathrm{~K}$ determined 


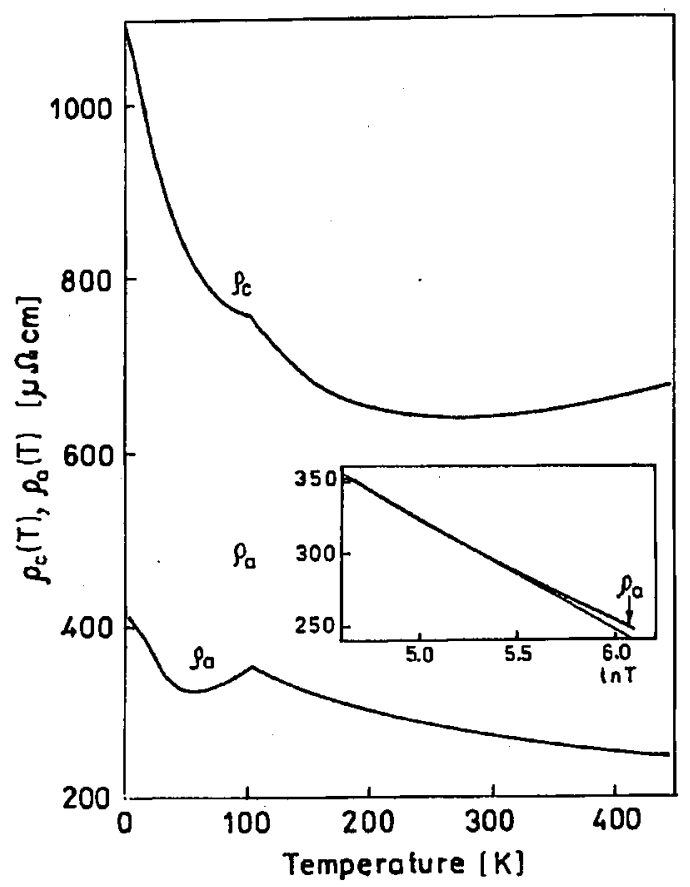

Fig. 1. Temperature dependence of the resistivity tensors along $a$-axis, $\rho_{a}$, and along $c$-axis, $\rho_{c}$. Inset shows almost linear dependence of $\rho_{a}$ on $\ln T$ in the paramagnetic range.

by previous $\rho_{a}$ examination [4] and with $T_{\mathrm{C}}=118 \mathrm{~K}$ shown by magnetization measurements of powder samples [6]. We think that the differences between the $T_{\mathrm{C}}$ are due to sample's stoichiometry variations.

The $\rho_{a}(T)$ dependence from Fig. 1 agrees well with that determined previously for paramagnetic phase [3] when temperature scale is renormalized by $T_{\mathrm{C}}$. The previous measurements were done by conventional four-point dc method on samples long enough to get precise data. They can be considered as some reference; approving our present results. They are reported here for the both tensors. The main features of the $\rho_{a}(T)$ and $\rho_{c}(T)$ behaviours are as follows:

(i) With increase in temperature in the paramagnetic range, there is only slight positive deviation of $\rho_{a}$ from the shown one in the inset logarithmic temperature dependence. At the same temperatures the $\rho_{c}$ deviate from the logarithmic dependence so strongly that slope of its temperature dependence becomes positive. Magnetic resistivity contributes to the total one much less for the tensor along $c$-axis than for tensor along $a$-axis.

(ii) Resistivity anomaly accompanying ferromagnetic phase transition is more pronounced for $\rho_{a}$ tensor than for $\rho_{c}$ one. Sharp change of slope of temperature dependence of resistivity from negative to positive and decrease in resistivity with 
decrease in $T$ (down to $0.5 T_{\mathrm{C}}$ ) are observed for the $\rho_{a}$ tensor. Small plateau is only seen on overall decrease in resistivity for the $\rho_{c}$ tensor.

(iii) Neither saturation nor activation behaviour of both resistivity tensors is seen in the lowest temperature range (down to $2 \mathrm{~K}$ ) when temperature decreases.

Bold line in Fig. 2 shows temperature dependence of the in-plane thermoelectric power, $S$. Its main features are: (i) $S$ is positive but respectively high as

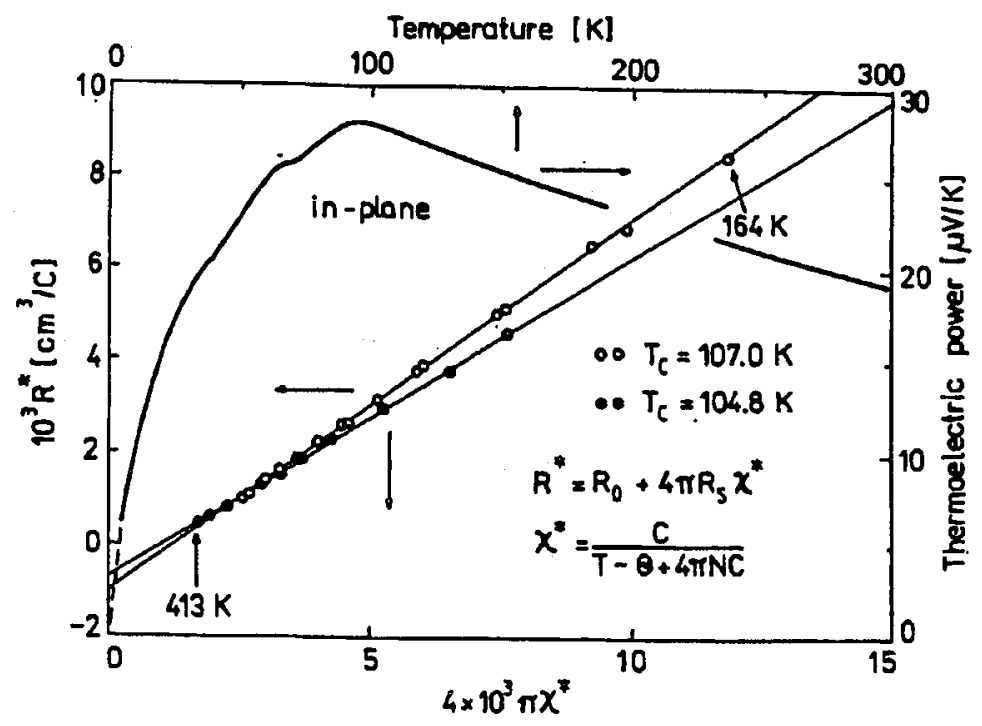

Fig. 2. Bold line shows temperature dependent thermoelectric power (maximum at $95 \mathrm{~K})$. Full and empty points present effective Hall coefficient, $R^{*}$, plotted versus effective susceptibility, $\kappa^{*}$, for two different crystals.

for normal metal case $\left(S^{\max }=+28 \mu \mathrm{V} / \mathrm{K}\right)$; (ii) it has poorly defined maximum in the vicinity of $T_{\mathrm{C}}$; (iii) the $S(T)$ curve is a convex line below and concave one above the maximum. The $S(T)$ dependence in the lowest temperatures is pointing to the origin of the coordinate system with a slope as high as $1.1 \mu \mathrm{V} / \mathrm{K}$.

Black and white points in Fig. 2 present data of the effective Hall coefficient $(H \| c), R^{*}$, plotted versus the effective susceptibility, $\kappa^{*}$, for samples whose Curie's temperature was equal to $104.8 \mathrm{~K}$ and $107.0 \mathrm{~K}$, respectively. Typical extrapolation procedure (shown in Fig. 2) for determination normal, $R_{0}$, and anomalous, $R_{\mathrm{s}}$, Hall coefficients was applied. Magnetic susceptibility for $c$ direction, $\kappa_{c}$, shows the Curie-Weiss behaviour [3]. Therefore, expression for the effective susceptibility $\kappa^{*}=\kappa_{c} /(1+4 \pi N C)$ is transformed to the form $\kappa^{*}=C /(T-\Theta+4 \pi N C)$ where $C$ and $\Theta$ are the Curie and Weiss constants, respectively and $N$ is demagnetizing factor. For fitted values of $\Theta-4 \pi N C$ (in order to get linear behaviour) we obtain $R_{0}\left(R_{\mathrm{s}}\right)$ values equal to $-1 \times 10^{-3}(0.81) \mathrm{cm}^{3} / \mathrm{C}$ and $-7 \times 10^{-4}(0.70) \mathrm{cm}^{3} / \mathrm{C}$ for white and black points, respectively. In the single-band model that corresponds to the carrier concentration of $(0.62-0.87) \times 10^{22}$ electrons per $\mathrm{cm}^{3}$ or $(0.41-0.58)$ electrons per formula unit. The positive value of thermopower and negative value 
of the normal Hall coefficient suggest that it is rather not a single-band system but the determined order of the carrier concentration may be correct. It means that UAsSe has metallic-like carrier concentration.

Short comment for these results is such that both temperature dependence of resistivity and its anisotropy cannot be understood in terms of the isotropic RKKY type exchange interaction. The UAsSe has the same crystal structure as $\mathrm{UAs}_{2}$ and similar to that of $\mathrm{USe}_{2}$. The in-plane resistivity for the $\mathrm{UAs}_{2}$ [7] shows typical magnetic metal behaviour. On the other hand, the resistivity along $c$-axis for the $\mathrm{US}_{2}$ [8] (presumably similar to $\mathrm{USe}_{2}$ ) shows activation behaviour at low temperatures. Intermediate character of the resistivity curves for UAsSe may show its intermediate electronic state. Taking into account high electron specific heat $\left(\gamma=41 \mathrm{~mJ} /\left(\right.\right.$ mole $\left.\left.^{2}\right)[9]\right)$, presence of band-like $f$-electrons (shown by Kerr-spectra [10]), negative slope of $\rho(T)$ and the thermoelectric power behaviour, the UAsSe may be classified as the Kondo lattice system on the heavy fermion side. High resistivity in the lowest temperatures and poorly pronounced resistivity and thermopower anomalies allow us to consider the system as being in the noncoherent state at low temperatures.

\section{References}

[1] Z. Fisk, P.C. Canfield, J.D. Thompson, M.F. Hundley, J. Alloys Comp. 181, 369 (1992).

[2] M. Kasaya, J. Phys. Soc. Jpn. 61, 3841 (1992).

[3] K.P. Belov, A.S. Dmitrievski, A. Zygmunt, R.Z. Levitin, W. Trzebiatowski, $Z h$. Eksp. Teor. Fiz. 64, 582 (1973).

[4] A. Wojakowski, Z. Henkie, Z. Kletowski, Phys. Status Solidi 14, 314 (1972).

[5] A. Wojakowski, Z. Henkie, Acta Phys. Pol. A 52, 401 (1977).

[6] A. Zygmunt, M. Duczmal, Phys. Status Solidi A 9, 113 (1972).

[7] Z. Henkie, Z. Kletowski, Acta Phys. Pol. A 42, 405 (1972).

[8] W. Suski, T. Gibiński, A. Wojakowski, A. Czopnik, Phys. Status Solidi A 9, 653 (1972).

[9] A. Blaise, R. Lagnier, A. Wojakowski, A. Zygmunt, M.J. Mortimer, J. Low Temp. Phys. 41, 61 (1980).

[10] W. Reim, J. Schoenes, F. Hulliger, Physica B 130, 64 (1985). 